\title{
An OFDMA Based Modem for PowerLine Communications over the Low Voltage Distribution Network
}

\author{
Sophie Gault*, Philippe Ciblat** and Walid Hachem* \\ * Supélec, Département Télécommunications, 91192 Gif-sur-Yvette Cedex, France \\ Phone: +331698514 54, Email: sophie.gault@supelec.fr \\ ** ENST, Département Comélec, 75634 Paris Cedex 13, France \\ Phone: +331458177 28, Email: philippe.ciblateenst.fr
}

\begin{abstract}
The paper describes an OFDMA based modem for multiple access PowerLine Communications (PLC) over the lowvoltage distribution network. Relying on a preliminary characterization of the French PLC network, a complete description of the modem is given. It concerns in particular the data frame structure, subcarrier allocation to users, the bitloading algorithm used for symbol coding, synchronization, and equalization. The proposed system is evaluated through simulations carried out using a simplified noise model.
\end{abstract}

\section{INTRODUCTION}

In recent years, in particular since the liberalization of the power and telecommunication markets, PowerLine Communications (PLC) have been the subject of an important research work. At the same time, the growing demand for multimedia communications provides a good prospect for PLC as a promising transmission technique for the "last mile" access network. In this configuration, the Low Voltage (LV) power grid is considered as a local loop. The connection to the Internet backbone is ensured at the Medium Voltage/Low Voltage (MV/LV) transformer, by a more conventional means, such as a fiber optic or a cable.

However, using electrical wires as a high speed communication channel remains a challenging idea, as the network structure and nature were not initially devised for that purpose. The main characteristics of the powerline channel seem quite unfavorable: multipath reflections induced by impedance mismatches, changes in the channel transfer function due to the switching of electrical devices, a highly diverse noise environment, and so forth. Therefore, an efficient transmission scheme has to include robust techniques in order to face the difficulties of such a medium and to get reliable and spectrally-efficient transmissions. In this paper, we focus on the design of a high speed PLC modem in the $[1 \mathrm{MHz}, 20 \mathrm{MHz}]$ band. This modem is based on an orthogonal frequency division multiplexing (OFDM) modulation, the considered multiple access scheme being the frequency division access OFDMA (Orthogonal Frequency Division Multiple Access). Up to the present day, OFDM has been adopted in many wireless systems as well

This work is supported by the French grant RNRT/IDILE: project partners are CEA/LETI, EDF (Électricité de France) R\&D, Elsys Design SA, ENST, INSA/IETR, Sagem SA, and Supélec. as in wireline applications, in particular for digital subscriber line (xDSL) services. Indeed, OFDM has many well known advantages: among others, a low complexity channel equalizer, a good resistance to narrowband and impulsive noise, and the possibility to approach the Shannon capacity by implementing a simple coding technique, the so-called bitloading technique. In the context of PLC, bitloading is appropriate because the channel is invariant for a period of time long in comparison with the OFDM symbol duration. However, OFDM systems also show a weakness in their sensitivity to synchronization errors.

The paper is organized as follows. Section II describes the characteristics of the network that are capital in the choice of the system parameters. In section III, we propose a data frame structure. Section IV and V provide a detailed description of the transmitter and the receiver. In section VI, simulation results are shown.

\section{NeTWORK DESCRIPTION}

In this section, we describe the communication link between the MV/LV transformer and the customer meter.

\section{A. Network Topology}

The LV power grid attracts considerable attention as a communication medium because it provides connection to every household. On average, an MV/LV transformer of the french EDF network feeds 200 households in an urban residential area ([1]). Therefore, seen from the MV/LV transformer, the network is a multiuser point-multipoint network.

The transmitted powerline signal experiences a considerable attenuation which increases with distance (that can reach a few hundred meters) but also with frequency ([2]). To deal with this problem, relay points where the signal is demodulated, regenerated and emitted again by modems called repeaters are introduced if need be. Therefore, a PLC local loop access network has a cell structure, similar to many radio networks. The communication system is thus multiuser and multicellular. We distinguish two types of cells. We call primary cell the unique cell where the Central Modem $(\mathbf{C M})$ at the MV/LV transformer plays the role of the base station, and the repeaters (R) play the role of terminal stations (see Fig. 1). Beside the 
repeaters, the primary cell also contains those of the customer modems $\mathbf{M}$ that are close to the modem CM. In Fig. 1, it is shown that repeaters also belong to secondary cells, in which they play the role of base stations that communicate with customer modems (M). A secondary cell will cover a repeater area, typically a building or a group of habitations. Following a configuration recommended by Electricité De France, we consider, in the sequel, a network with $Q=16$ repeaters, each repeater transmitting toward 16 modems. Consequently 256 users can be connected to the modem CM. Fig. 1 brings to the fore a bus-connected architecture since all types of modems are branched in parallel on the single-phase line.

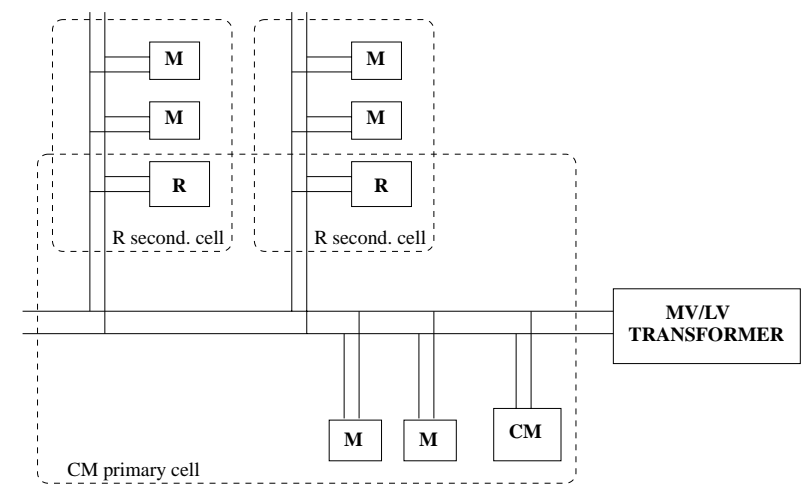

Fig. 1. A PLC cellular network

\section{B. Channel Characteristics}

The frequency band $[1 \mathrm{MHz}, 20 \mathrm{MHz}]$ can support the high rates needed by many Internet applications. Note that the bandwidth is the same as in VDSL ([3]). Channel transfer function between any two modems in the network is that of a complicated transmission line with many stubs having terminated loads of various impedances. Because of the impedance mismatches, the channel is characterized by multipath effect and a long time dispersion equal to a few microseconds. This delay spread is long compared to the inverse of the (cumulative) symbol rate which is almost the sampling period $\left(T_{s}=0.05 \mu s\right.$, see section IV-A). In Fig. 2, we plot the realization of a channel impulse response measured by Electricité de France in downlink from a household distant of 20 meters to the MV/LV transformer in a residential area.

Furthermore, this impulse response changes when electrical

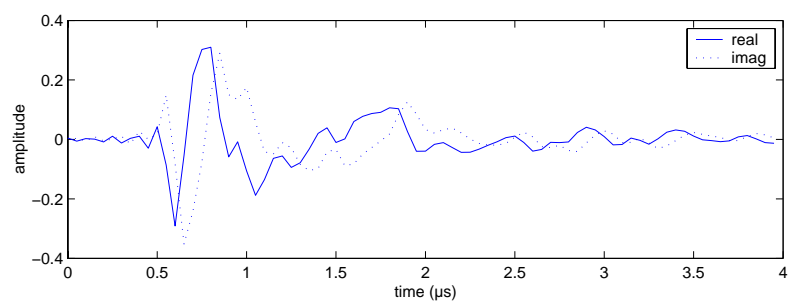

Fig. 2. A powerline channel impulse response.

devices are switched on or off. It appears that one can consider it as being invariant for a period of the order of a few seconds. On this basis, training sequences have to be inserted periodically to re-estimate the channel in both uplink and downlink directions. Because this period is rather long, it is perfectly conceivable to transmit the Channel State Information (CSI) toward the transmitter via a feedback channel, and to exploit judiciously this CSI.

\section{FRAME STRUCTURE}

The signals exchanged in the primary cell are separated from those of the secondary cells using Time Division. Furthermore, in each cell, the downlink is separated from the uplink using Time Division Duplexing (TDD). The frame structure is illustrated Fig. 3. Each frame is divided into 4 consecutive time slots corresponding to the 4 possibilities: the slots CMD, CMU, RD, and RU correspond to the primary cell in downlink, primary cell in uplink, secondary cell in downlink, and secondary cell in uplink respectively. The relative durations of these slots can be adjusted according to the data rate requirements of each of the corresponding four transmission modes. The whole frame contains 256 OFDM symbols. Every 64 frames are grouped into a superframe. As

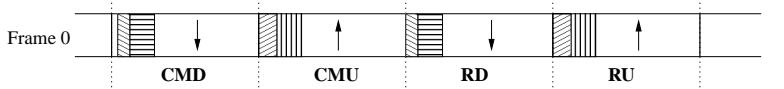

\begin{tabular}{|c|c|c|c|c|c|}
\hline \multirow{2}{*}{$\begin{array}{l}\text { Frame i } \\
(i \neq 0)\end{array}$} & $\downarrow$ & $\uparrow$ & $\downarrow$ & $\uparrow$ & \\
\hline & CMD & CMU & RD & RU & \\
\hline ris & \multicolumn{5}{|c|}{ Training sequence $\mathbf{A}$ dedicated to DL channel estimation, cell search (in frame 0 ) and synchronization } \\
\hline 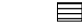 & \multicolumn{5}{|c|}{ Training sequence $\mathbf{P N}$ dedicated to DL noise-plus-interference estimation (in frame 0) } \\
\hline 泽 & \multicolumn{5}{|c|}{ Training sequence $\mathbf{A}^{\prime}$ dedicated to UL channels estimation (in frame 0 ) } \\
\hline 盂 & \multicolumn{5}{|c|}{ Training sequence $\mathbf{P N}$ ' dedicated to UL noise-plus-interference estimation (in frame 0 ) } \\
\hline
\end{tabular}

Fig. 3. Frames based on TDD slots

the duration of an OFDM symbol is $T_{\text {OFDM }}=117,4 \mu \mathrm{s}$ (see section IV-A), the duration of a superframe is equal to $64 \times 256 \times T_{\text {OFDM }}=1.92 \mathrm{~s}$, a time period over which the channel is considered as invariant. The first frame in a superframe, denoted in Fig. 3 as frame 0, has a special structure. A modem that has the intention to access the network will use the OFDM symbols denoted by $\mathbf{A}$ at the beginning of frame 0 to estimate the (downlink) channels coming from the different repeaters. Symbols PN in this frame are used to estimate the Power Spectral Density (PSD) of the noise plus interference. Based on these estimations, this modem will select the most appropriate cell. Because the size $N$ of the discrete Fourier transform (DFT) or equivalently, the number of subcarriers in an OFDM symbol is large $(N=2048$, see section IV-A), the frequency of the transmitter sampling clock has to be estimated. This is also one purpose of OFDM symbols A. Because the receiver clock has to remain locked on the transmitter clock, symbols $\mathbf{A}$ are sent again in the downlink slots of the frames other than frame 0. Frequency clock estimation is done only in the downlink and all modems in a cell will have their sampling clocks synchronized on the clock of their base station. As a consequence, the whole network is synchronized on the clock of the modem $\mathbf{C M}$. 
The base station of a cell has to estimate the uplink channels as well as the noise plus interference PSD. In the uplink slots of frame 0 , the modems in a cell send training sequences toward their base station for that purpose.

Related algorithms are detailed in section V.

\section{Modem : Transmitter Side}

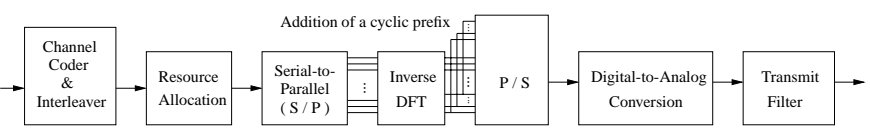

Fig. 4. Transmitter block diagram

\section{A. OFDM Modulation Design}

Table I gathers the system main parameters.

\section{TABLE I}

OUR POWERLINE SYSTEM PARAMETERS

\begin{tabular}{l|c}
\hline Sampling period $\left(T_{s}\right)$ & $0.05 \mu s$ \\
\hline Number of sub-carriers $(N)$ & 2048 \\
\hline Channel delay spread & $5 \mu s=100 T_{s}$ \\
\hline Guard interval duration & $15 \mu s=300 T_{s}$ \\
\hline DFT block duration & $102,4 \mu s=2048 T_{s}$ \\
\hline OFDM symbol duration $\left(T_{\text {OFDM }}\right)$ & $117,4 \mu s=2348 T_{s}$ \\
\hline
\end{tabular}

The sampling frequency $F_{s}$ is $20 \mathrm{MHz}$. The guard interval has been fixed to $D=300$ samples. It has been dimensioned so as to absorb all the transmission delays in a cell, which ensures synchronization at the level of OFDM symbols. In order to limit the loss of spectral efficiency induced by the guard interval, the number of subcarriers has to be chosen large: $N=2048$, and this reduces the loss of spectral efficiency $\eta=D /(N+D)$ to $\eta=12.8 \%$.

\section{B. Dynamic Bit Allocation and Coding}

Let $K$ be the number of users in a given cell. Assume that subcarrier $n$ has been allocated to user $k$, and denote by $\operatorname{SNIR}_{k, n}$ the associated Signal to Noise plus Interference Ratio (SNIR). Notice that in our case, the interference is the undesired signal that comes from nearby cells, as explicitly detailed in section V-C. Given this SNIR, we have to choose the greatest symbol constellation size compatible with a target Bit Error Rate (BER). This is the well known bitloading principle. The chosen constellation will be characterized by a number of bits per symbol $b_{k, n}$ given by:

$$
b_{k, n}=\left\lfloor\log _{2}\left(1+\mathrm{SNIR}_{k, n} / \Gamma_{k}\right)\right\rfloor
$$

where $\lfloor$.$\rfloor stands for rounding down to the nearest integer and$ $\Gamma_{k}$ is the so-called Signal to Noise Ratio Gap ([4]). Assuming a hard decoding at the output of the constellation decoder, this gap depends on the target BER at the output of the hard decoder. For instance, if we require the BER at the output of the hard decoder to be equal to $10^{-3}$, then $\Gamma_{k}=6 \mathrm{~dB}$. Considering the Forward Error Correction (FEC), we selected the standard (240, 224) octet-oriented Reed-Solomon (RS) code followed by an octet-oriented interleaver. This coding scheme is similar to the one described in the VDSL norm ([3]). Notice that the noise environment is similar in the two contexts of PLC and VDSL. In particular, in addition to Gaussian noise, impulse noise is present in both situations. RS codes are known to combat the effect of these noises efficiently. Notice also that (1) is usually considered as reliable when the noise is Gaussian. In our situation, the noise has a non Gaussian part due to intercell interference. However, for a BER equal to $10^{-3}$ at the output of the hard decoder (resulting in a BER of $10^{-7}$ at the output of the RS decoder), it appears that (1) can still be used in our context.

\section{Subcarrier Allocation to Users}

We present here a very simple OFDMA algorithm for allocating the subcarriers to users within a cell. It is an adaptation of the one proposed by Rhee and Cioffi in [5], where they assume equivalent users rate requirements.

Let $\left(R_{k}\right)_{k=1, \ldots, K}$ be the rates given to the $K$ users. Define the subcarrier allocation function as $\mathcal{I}(n)=k$ if subcarrier $n$ is given to user $k$. The allocation algorithm is :

1) Init:

$$
\begin{aligned}
& A=\{0, \cdots, N-1\}, \quad B=\{0, \cdots, K-1\}, \\
& c_{k, n}=\left\lfloor\log \left(1+\operatorname{SNIR}_{k, n} / \Gamma_{k}\right)\right\rfloor \forall k \in B \quad \forall n \in A
\end{aligned}
$$

2) For $k=0, \cdots, K-1\{$

$$
\begin{aligned}
& M=\max _{j \in A}\left(c_{k, j}\right) \\
& \text { If } M \neq 0 \\
& \text { Find } E: c_{k, j}=M \quad \forall j \in E \\
& \text { Find } n: c_{i, n}=\min _{j \in E}\left(\max _{i \neq k} c_{i, j}\right) \\
& R_{k}=M, A=A-\{n\}, \mathcal{I}(n)=k \\
& \text { else } \\
& B=B-\{k\}\}
\end{aligned}
$$

(c)

3) While $A \neq \emptyset$ and $B \neq \emptyset\{$

(a)

(c)

$$
\text { Find } k: R_{k}=\min _{i \in B} R_{i}
$$$$
M=\max _{j \in A}\left(c_{k, j}\right)
$$

$$
\text { If } M \neq 0
$$$$
\text { Find } E: c_{k, j}=M \forall j \in E
$$

Find $n: c_{i, n}=\min _{j \in E}\left(\max _{i \neq k} c_{i, j}\right)$

$$
R_{k}=R_{k}+M, A=A-\{n\}, \mathcal{I}(n)=k
$$$$
\text { else }
$$$$
B=B-\{k\}\}
$$

Let us now explain the main differences w.r.t. Rhee and Cioffi's algorithm. Rhee and Cioffi simply use the SNIR as a unique distinction criterion. However, there is a truncation effect due to the use of the floor function in (1), since subcarriers having different SNIRs can transmit the same bitrate. Therefore, the subcarrier uppermost assigned to a given user is chosen among a set of subcarriers with SNIRs leading to the same maximal transmitted bitrate. Then, on each subcarrier belonging to this set, one finds the maximum among the bitrates all other users would reach in a single user context, and the subcarrier corresponding to the minimum of all these maxima is allocated, so as to penalize as less as possible other users. Moreover, a user is discarded (i.e. he will not be considered in the following of algorithm) as soon 
as the number of bits supported by its "best" subcarrier is no longer strictly positive.

\section{Modem : RECEIVER SIDE}

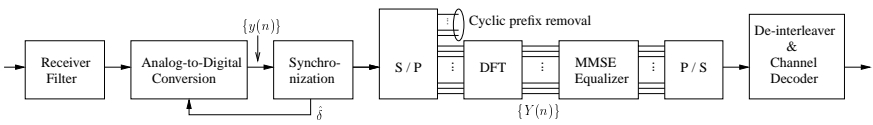

Fig. 5. Receiver block diagram

\section{A. Synchronization and Channel Estimation}

A modem with the intention to access the network will try to detect the CMD or the RD slots of the frame 0 in a superframe. The procedure it has to follow is described by Fig. 6 .

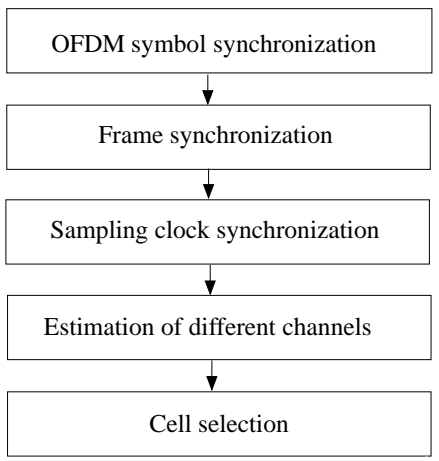

Fig. 6. Synchronization procedure

First, the modem has to find the symbol timing, i.e. the beginning of the OFDM symbols, in order to position accurately the Discrete Fourier Transform (DFT) window. To that purpose, the OFDM symbol periodical structure is exploited by computing a sliding $D$-sample correlation of the received signal with its replica shifted back by $N$ samples ([6]):

$$
R(n)=\frac{\sum_{k=0}^{D-1} y^{*}(n+k) y(n+k+N)}{\sum_{k=0}^{D-1}|y(n+k+N)|^{2}}
$$

where $\{y(n)\}$ are the received signal samples in time domain. Simple threshold detection is then implemented to locate the correlation peaks, i.e. the OFDM symbols beginning.

In a second step, the modem has to locate the frames. This can be done by detecting symbols $\mathbf{A}$ which have a particular structure. Indeed, in symbols A, all subcarriers with odd ranks are put to zero, and a pseudo-noise sequence is transmitted on even subcarriers. Thus if we compute the ratio of the sum of the powers received on even subcarriers to sum of the powers received on the odd subcarriers, peaks will appear every time a symbol $\mathbf{A}$ is received, i.e., at the beginning of slots $\mathbf{C M D}$ and RD. The symbol A detection function writes:

$$
S(n)=\frac{\sum_{k=0}^{(N-1) / 2}\left|Y_{n}(2 k)\right|^{2}}{\sum_{k=0}^{(N-1) / 2}\left|Y_{n}(2 k+1)\right|^{2}}
$$

where $Y_{n}(l)$ represents the signal received on subcarrier $l$ of OFDM symbol $n$.
Since the number of subcarriers in an OFDM symbol is large, the frequency of the transmitter sampling clock has to be estimated. Equivalently, denoting by $T_{s}$ the transmitter clock period, the receiver clock period in its free oscillation mode can be written $T_{s}^{\text {free }}=(1+\delta) T_{s}$. The mismatch parameter $\delta$ has to be estimated and to do this, the particular structure of symbol A can be exploited again. As the even subcarriers are the only active subcarriers, a transmitted A symbol consists in two consecutive identical halves in the time domain ([6]). At the receiver, a DFT operation of size $N / 2$ is sequentially performed on each of these two halves. Denoting by $\left(\tilde{Y}_{1}(0), \ldots, \tilde{Y}_{1}(N / 2-1)\right)$ and $\left(\tilde{Y}_{2}(0), \ldots, \tilde{Y}_{2}(N / 2-1)\right)$ the outputs of these two DFTs respectively, an estimate $\hat{\delta}$ of $\delta$ can be ([7]) :

$$
\hat{\delta}=\frac{1}{2 \pi} \frac{\sum_{l=0}^{N / 2-1} l \Im\left(\tilde{Y}_{2}(l) \tilde{Y}_{1}^{*}(l)\right)}{\sum_{l=0}^{N / 2-1} l^{2} \Re\left(\tilde{Y}_{2}(l) \tilde{Y}_{1}^{*}(l)\right)}
$$

We now turn to the channel estimation and consider for example the situation where a modem $\mathbf{M}$ receives signals from a certain number of repeaters. The pseudo-noise training sequence sent on symbols $\mathbf{A}$ by a repeater is specific to this repeater, and these sequences are decorrelated. Therefore, the different channels can be estimated by the modem $\mathbf{M}$ by correlation of the signal it receives in the time domain with these training sequences. The cell that corresponds to the estimated impulse response vector with the largest Euclidean norm is finally selected by modem $\mathbf{M}$.

\section{B. MMSE equalization}

Let the same $k^{t h}$ user transmitting on the $n^{t h}$ subcarrier belong to the cell 1 . The MMSE detection of a QAM symbol emitted by the repeater writes :

$$
\tilde{S}_{k, n}^{(1)}=\frac{\sqrt{Z_{n}} H_{k, n}^{(1)}{ }^{*}}{Z_{n}\left|H_{k, n}^{(1)}\right|^{2}+\sum_{q=2}^{Q} Z_{n}\left|H_{k, n}^{(q, 1)}\right|^{2}+\sigma_{k, n}^{2}} Y_{k, n}^{(1)}
$$

where $Z_{n}$ is the QAM symbol energy. This energy is constrained by a PSD mask to avoid radiated emission from PLC on other services using the same bandwidth, such as amateur radio operators. $H_{k, n}^{(1)}$ and $H_{k, n}^{(q, 1)}$ are respectively the coefficient of the channel between the repeater of the cell 1 and its $k^{t h}$ modem on the $n^{t h}$ subcarrier, and the coefficient of the channel between the repeater of the cell $q$ and the same modem on the same subcarrier.

\section{Noise-plus-Interference estimation}

Still considering the same user, once his channel coefficients are known, the noise-plus-interference denoted $\mathrm{NI}_{k, n}^{(1)}$ remains to be estimated on every subcarrier, as required for the bitloading algorithm and the MMSE detection.

$$
\mathrm{NI}_{k, n}^{(1)}=\sum_{q=2}^{Q} Z_{n}\left|H_{k, n}^{(q, 1)}\right|^{2}+\sigma_{k, n}^{2}
$$

One can notice there is no need to estimate separately the interfering channels and the noise variance. In downlink, a simple solution based on the symbols PN exists. At the 
begining of the superframe, after the symbol $\mathbf{A}$, each repeater emits $Q-1 \mathbf{P N}$ symbols and one null symbol. The localization of the null symbol follows the rule according to which when one repeater emits its null symbol, all other repeaters are transmitting their non-null PN symbols. As the power emitted for the $\mathbf{P N}$ symbols is equivalent to the power emitted in data mode, the signal received by the modem during the silence of one repeater will provide an estimate of the sum of the intercell interference of the other repeaters with the AWGN. This enables us to estimate exactly the noise-plus-interference variance. And finally, for user $k$ transmitting on subcarrier $n$ in the cell 1, the Signal-to-Noise-plus-Interference has the following expression:

$$
\operatorname{SNIR}_{k, n}^{(1)}=\frac{Z_{n}\left|H_{k, n}^{(1)}\right|^{2}}{\mathrm{NI}_{k, n}^{(1)}}
$$

In uplink, all modems in all cells emit $Q-1$ PN' symbols and one null symbol. The localization of the null symbol is the same for all modems inner to a same cell. And when the modems of a particular cell emit their null symbol, they estimate the intercell interference plus the AWGN because all other modems are transmitting their non-null PN' symbols. Therefore, the estimated interference power is worse than in data mode since the subcarriers have not been allocated yet. All signals emitted simultaneously by all modems on all subcarriers are taken into account. Notice that this results in a pessimistic uplink noise-plus-interference estimate.

\section{RESUlts}

Simulations were carried out using the parameters described in section IV-A. The channels used, provided by "Électricité de France", have been measured in a residential area of Paris southern suburbs, and a simplified noise model (with only AWGN) is assumed. The initial sampling clock offset introduced is $30 \mathrm{ppm}$. The synchronization, channel estimation, and noise estimation algorithms are simulated, with a RS-coder and encoder, but no interleaver. The targeted BER after RSdecoding is $10^{-7}$.

In Fig. 7 and 8, we plot bitrates vs. the $E_{s} / N_{0}$ in $\mathrm{dB}$, which

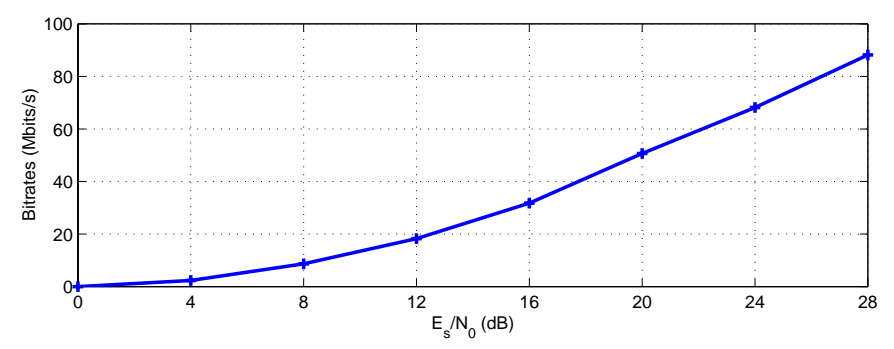

Fig. 7. Bitrates vs. $E_{s} / N_{0}$ in a monocellular single user context

is defined as the ratio of the energy emitted per symbol to the noise variance (the AWGN is taken into account but not the intercell interference). In Fig. 7, we consider only one modem in one cell. For $E_{s} / N_{0}=17 \mathrm{~dB}$, a bit rate of 38 Mbits/s is achieved which corresponds to a spectral efficiency of $2 \mathrm{bits} / \mathrm{s} / \mathrm{Hz}$. Notice that more sophisticated FEC encoding
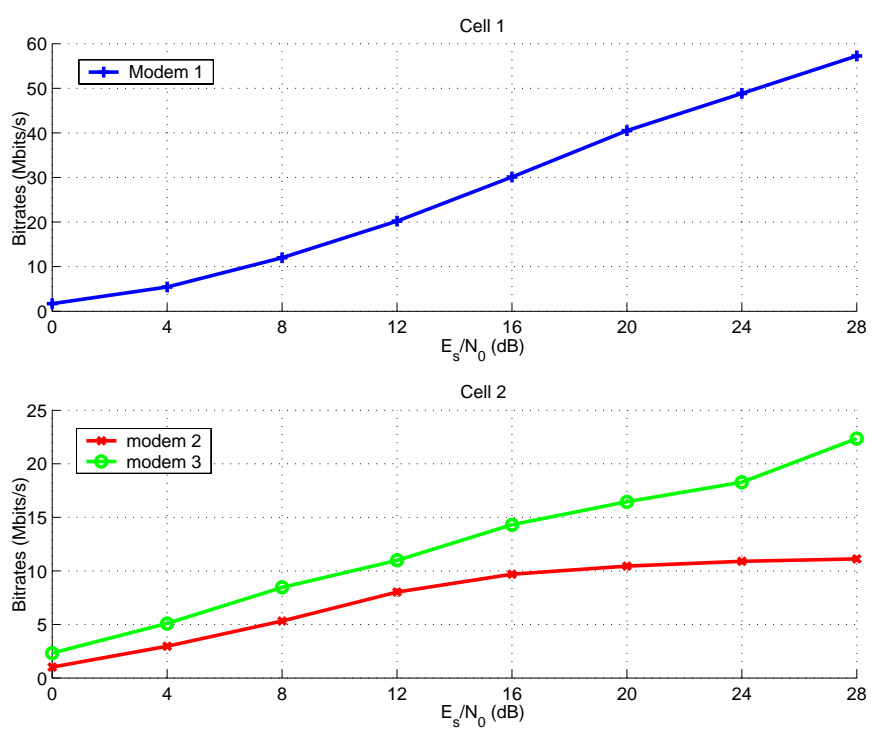

Fig. 8. Bitrates vs. $E_{s} / N_{0}$ in a multicellular multiuser context

and decoding techniques can be used to reduce the margin $\Gamma_{k}$ and then to increase the spectral efficiency. In Fig. 8, three users are located in two cells. So this simulation also includes the cell selection and the subcarrier allocation algorithms. As a result, the modem 1 belongs to cell 1 while the modems 2 and 3 belong to cell 2 . In reality, the distance between the modem 1 and the cell 1 repeater is $10 \mathrm{~m}$. In the cell 2 , the distances between the modem 2 and the repeater and between the modem 3 and the repeater are $160 \mathrm{~m}$ and $30 \mathrm{~m}$ respectively. Finally the distance between the two repeaters is about $20 \mathrm{~m}$ which corresponds to a standard distance between two buildings. The bitrate degradation in cell 1 (33 Mbits/s) w.r.t. the single cell case is due to intercell interference which gives lower SNIRs. In the second cell, the two modems obtain relatively fair bitrates in spite of the distance of modem 2 : $10 \mathrm{Mbits} / \mathrm{s}$ for modem 2 and $15 \mathrm{Mbits} / \mathrm{s}$ for modem 3.

The algorithms for symbol synchronization, frame synchronization, and cell selection offer very good results (in practice, a detection rate of $100 \%$ is reached).

\section{REFERENCES}

[1] Open PLC European Research Alliance (OPERA), http://www.istopera.org/.

[2] M. Zimmermann and K. Dostert, "A multipath signal propagation model for the powerline channel in the high frequency range," in International Symposium on Power-Line Communications (ISPLC), 1999.

[3] ETSI, Transmission and Multiplexing (TM); Access transmission systems on metallic access cables; Very high speed Digital Subscriber Line (VDSL); Part 2: Transceiver specification, 2002-2002, TS 101 270-2.

[4] J.M. Cioffi, A Multicarrier Primer, Technical Report, Stanford University, http://www-isl.stanford.edu/people/cioffi/pdf/multicarrier.pdf.

[5] W. Rhee and J.M. Cioffi, "Increase in capacity of multiuser OFDM system using dynamic subchannel allocation," in IEEE Vehicular Technology Conference (VTC), 2000.

[6] T.M. Schmidl and D.C. Cox, "Robust Frequency and Timing Synchronization for OFDM," IEEE Trans. on Communications, vol. 45, no. 12, pp. 1613-1621, Dec. 1997.

[7] S. Gault, W. Hachem, and P. Ciblat, "Joint sampling clock offset and channel estimation for OFDM signals: CRB and algorithms," submitted to IEEE Trans. on Signal Processing, revised in November 2004. 